08

\title{
Процессы текстурообразования в тонкослойной системе $\mathrm{Ni}-\mathrm{W} / \mathrm{TiN}$
}

\author{
() М.С. Сунгуров, В.А. Финкель \\ Национальный научный центр „Харьковский фризико-технический институт“, \\ 61108 Харьков, Украина \\ e-mail: sungurovm@kipt.kharkov.ua
}

(Поступило в Редакцию 29 марта 2016 г. В окончательной редакции 25 января 2018 г.)

Получены тонкопленочные двухслойные композиции с TiN покрытием на основе ферромагнитного сплава $\mathrm{Ni}-5$ at.\% W и парамагнитного сплава $\mathrm{Ni}-9.5$ at.\% W. Методами рентгеноструктурного анализа изучены процессы текстурообразования в обеих компонентах системы Ni-W/TiN. Установлено, что в ленте из $\mathrm{Ni}-9.5$ at.\% W происходит переориентация кристаллических плоскостей под воздействием покрытия, ведущая к существенному усилению кубической текстуры подложки. Показано, что при определенных условиях нанесения тонкослойного покрытия в системе $\mathrm{Ni}-9.5$ at.\% W/TiN имеет место формирование квазимонокристаллических слоев TiN с кубической текстурой.

DOI: 10.21883/JTF.2018.08.46312.1824

\section{Введение}

Как известно, создание немагнитных металлических лент с кубической текстурой является одним из важнейших путей получения оптимальных подложек для высокотемпературных сверхпроводников второго поколения (2G HTS) по технологии RABiTS (Rolling Assisted Biaxially Textured Substrates) [1-4].

„Архитектура“ 2G HTS состоит как минимум из трех основных компонент:

1) основа (металлическая лента, преимущественно из сплавов $\mathrm{Ni}-\mathrm{W}$ [5-7], или Hastelloy [8]);

2) буферный слой (широкое применение находят такие соединения, как $\mathrm{CeO}_{2}$ и $\mathrm{MgO}[9,10]$; отдельно стоит упомянуть [11-13] $\mathrm{TiN}$ - перспективный материал, обладающий широким спектром физических свойств $[14,15]$, необходимых для построения эффективной архитектуры $2 \mathrm{G}$ HTS, а именно высокой термостойкостью, повышенной твердостью, стабильностью во влажных условиях (в отличие от гигроскопичного $\mathrm{MgO}$ ) и т.д.);

3) тонкопленочное сверхпроводящее покрытие (в качестве сверхпроводника используют исключительно $\mathrm{REBCO}$ соединения, такие как $\mathrm{YBa}_{2} \mathrm{Cu}_{3} \mathrm{O}_{7-\delta}$ (YBCO) c критической температурой $T_{c} \sim 92 \mathrm{~K}$ ).

Величина транспортного тока, протекающего без диссипаций по $2 \mathrm{G}$ HTS проводнику, во многом зависит от кристаллической структуры, магнитных и механических свойств металлической подложки, на которую наносятся слои буферного покрытия и высокотемпературного сверхпроводника (HTS) [16]. Для обеспечения высокой токонесущей способности на уровне критической плотности тока $j_{c} \sim 10^{5}-10^{6} \mathrm{~A} / \mathrm{cm}^{2}$ при температуре кипения жидкого азота подложка должна: 1) обладать совершенной кубической текстурой типа $\{100\}\langle 001\rangle$, необходимой для реализации эпитаксиального роста слоя HTS по схеме: $\{100\}_{\mathrm{Ni}-\mathrm{W}}\left\|\{100\}_{\text {buffer layer }}\right\|\{100\}_{\mathrm{YBaCuO}}$; 2) находиться в парамагнитном состоянии при низких температурах для снижения уровня ферромагнитных потерь $[17,18]$.

На сегодняшний день в качестве наиболее перспективных материалов для изготовления базового элемента в архитектуре 2G HTS принято рассматривать ГЦК сплавы системы $\mathrm{Ni}-\mathrm{W}$. Однако проблема получения подложек, сочетающих заданные свойства (см. выше), до конца не решена. Для ленты из сплава $\mathrm{Ni}-5$ at.\% W, наряду с формированием кубической текстуры рекристаллизации, характерно наличие коллинеарной ферромагнитной структуры. В результате повышения концентрации легирующего компонента кристаллическая решетка сплава расширяется, понижается степень обменного взаимодействия $\mathrm{Ni}-\mathrm{Ni}$, и ферромагнитное состояние нарушается - сплавы с содержанием вольфрама $\geq \sim 9.5$ at.\% находятся в парамагнитном состоянии во всем диапазоне температур [19]. Однако рост концентрации $W$ приводит к снижению энергии дефектов упаковки $E_{s f}$, что в свою очередь препятствует образованию кубической текстуры после высокотемпературного отжига [20,21].

Ранее [22-25] при исследовании возможности получения кубической текстуры в парамагнитных сплавах $\mathrm{Ni}-\mathrm{W}$ были обнаружены структурные эффекты, дающие основание полагать, что нанесение на ленту из сплава Ni-9.5 at.\% W тонких слоев TiN при определенных условиях может способствовать развитию кубической текстуры в ленте-подложке.

В связи с изложенным выше целью настоящей работы является изучение природы и механизмов образования кубической текстуры в обеих компонентах тонкослойной системы $\mathrm{Ni}-\mathrm{W} / \mathrm{TiN}$. Можно полагать, что решение этой, фундаментальной по своей сути задачи физики твердого тела, открывает перспективы для отыскания решения актуальной прикладной проблемы современной техники - разработки принципов получения композиционных материалов типа $\mathrm{Ni}-\mathrm{W} / \mathrm{TiN}$ на основе парамагнитного сплава $\mathrm{Ni}-9.5$ at.\% W для обеспечения максимальной токонесущей способности $2 \mathrm{G}$ HTS сверхпроводников. 
Для достижения цели настоящей работы реализована следующая программа исследований:

1. Получение и аттестация сплавов $\mathrm{Ni}-\mathrm{W}$ различного состава и лент на их основе.

2. Выбор режимов механической и термической обработки лент, а также исследование их свойств с целью выбора достаточно представительных объектов для проведения исследований.

3. Оптимизация условий ионно-плазменного осаждения буферных слоев TiN на поверхность лент из сплавов $\mathrm{Ni}-\mathrm{W}$.

4. Проведение рентгеноструктурных исследований на всех стадиях получения и обработки образцов. Кроме изучения общего характера дифракционной картины объектов исследования необходимо было изучить:

4.1. Особенности текстурообразования в обеих компонентах тонкопленочной композиции $\mathrm{Ni}-\mathrm{W} / \mathrm{TiN}$.

4.2. Зависимости параметров кристаллической решетки обеих компонент тонкопленочной системы $\mathrm{Ni}-\mathrm{W} / \mathrm{TiN}$ от толщины покрытия для установления роли напряжений в процессе формирования текстуры в подложке из сплавов $\mathrm{Ni}-\mathrm{W}$ и слое $\mathrm{TiN}$.

\section{1. Методика эксперимента}

В настоящем разделе представлены методические аспекты исследования, которые включают: синтез сплавов $\mathrm{Ni}-\mathrm{W}$ (разд. 1.1.), получение на их основе металлических лент (разд. 1.2), нанесение на ленты-подложки покрытий нитрида титана TiN (разд. 1.3), определение толщины слоя TiN (разд. 1.4), анализ текстуры в системе $\mathrm{Ni}-\mathrm{W} / \mathrm{TiN}$ (разд. 1.5).

\section{1. Синтез сплавов $\mathrm{Ni}-\mathrm{W}$}

В качестве исходных материалов использовали порошки $\mathrm{Ni}$ и W, номинальная чистота которых составляла 99.98-99.99\% по металлическим примесям. Для очистки от газовых примесей, как правило, присутствующих в продуктах промышленного производства, порошки подвергались термической обработке: отжиг порошков $\mathrm{Ni}$ при температуре $T=850^{\circ} \mathrm{C}$ в вакууме и высокотемпературный отжиг $\mathrm{W}$ при $T=1000-1200^{\circ} \mathrm{C}$ в восстановительной среде $\mathrm{Ar}+4 \% \mathrm{H}_{2}$ [26].

Сплавы $\mathrm{Ni}-\mathrm{W}$ различного состава получали методом порошковой металлургии. Исходные компоненты тщательно перемешивались с помощью мельницыступки (Pulverizette) в необходимых пропорциях $(0-9.5$ at.\% W). Масса смеси составляла $m \sim 20$ g. Затем порошковую смесь прессовали с помощью стандартного лабораторного пресса в виде параллелепипедов в целях оптимизации последующей механической обработки полученных заготовок. Образцы спекали в высоком вакууме $\left(p \sim 10^{-6}-10^{-5}\right.$ Torr $)$ при температуре $T=1250^{\circ} \mathrm{C}$ в течение $t=4 \mathrm{~h}$.

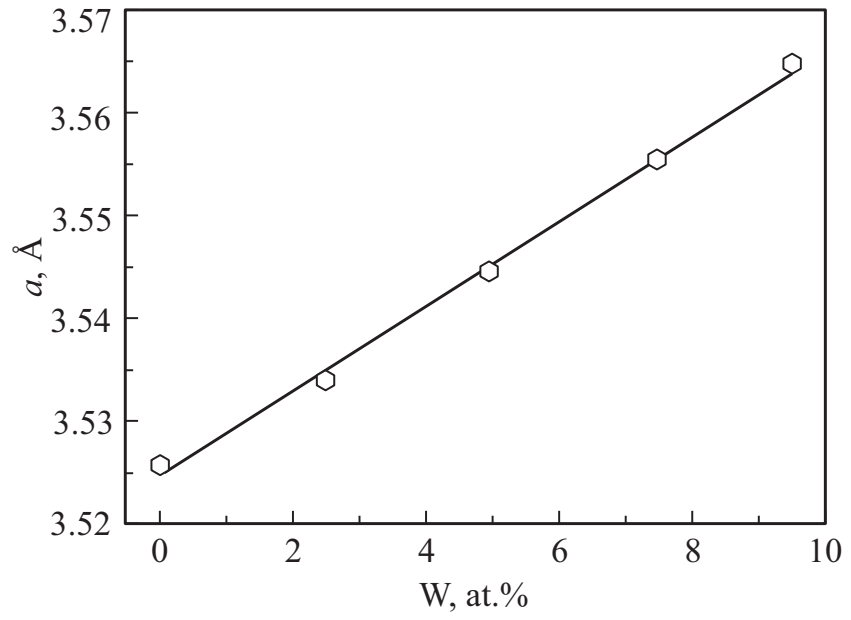

Рис. 1. Зависимость параметров решетки от концентрации вольфрама в сплаве $\mathrm{Ni}-\mathrm{W}$ различного состава.

Для аттестации образцов применяли методы рентгеноструктурного анализа (Дрон УМ-1, $\mathrm{Cu} K_{\alpha 1}$-излучение). На рентгенограммах линий, отличных от ГЦК фаз, не наблюдалось. На рис. 1 показана зависимость параметров решетки $a_{\mathrm{Ni}-\mathrm{W}}$ от концентрации вольфрама в сплаве. Зависимость носит практически линейный характер, что свидетельствует о полном соответствии состава сплавов заданным значениям концентрации.

\section{2. Получение ленты из сплавов $\mathrm{Ni}-\mathrm{W}$}

Полученные $\mathrm{Ni}-\mathrm{W}$ заготовки подвергались механической и термической обработке для изготовления лентподложек. Установлено, что оптимальным условием механической обработки сплавов $\mathrm{Ni}-\mathrm{W}$ с высоким содержанием вольфрама является холодная прокатка со степенью обжатия порядка 95\% без проведения промежуточных отжигов. Идея [22] использования подобной методики заключается в том, что наличие в ленте напряжений, приобретенных в процессе холодной деформации образца, может способствовать формированию кубической текстуры рекристаллизации за счет повышения $E_{s f}$.

Характерные размеры образца под прокатку составляли $10 \times 50 \times 2 \mathrm{~mm}$, а конечная толщина ленты равнялась $d \sim 100 \mu \mathrm{m}$.

Заключительной операцией в процессе получения подложки являлся высокотемпературный отжиг в вакууме при $T=1100^{\circ} \mathrm{C}$ и $t=2 \mathrm{~h}$.

Для оптимизации процесса получения ленточных текстурированных образцов необходимо было получить информацию о механических свойствах лент из сплавов $\mathrm{Ni}-\mathrm{W}$ различного состава. На рис. 2 приведены зависимости предела текучести $\sigma_{f}$ и предела прочности $\sigma_{b}$ от концентрации вольфрама в сплаве, указывающие на эффекты повышения $\sigma_{f}$ и $\sigma_{b}$ с ростом содержания легирующей примеси. 

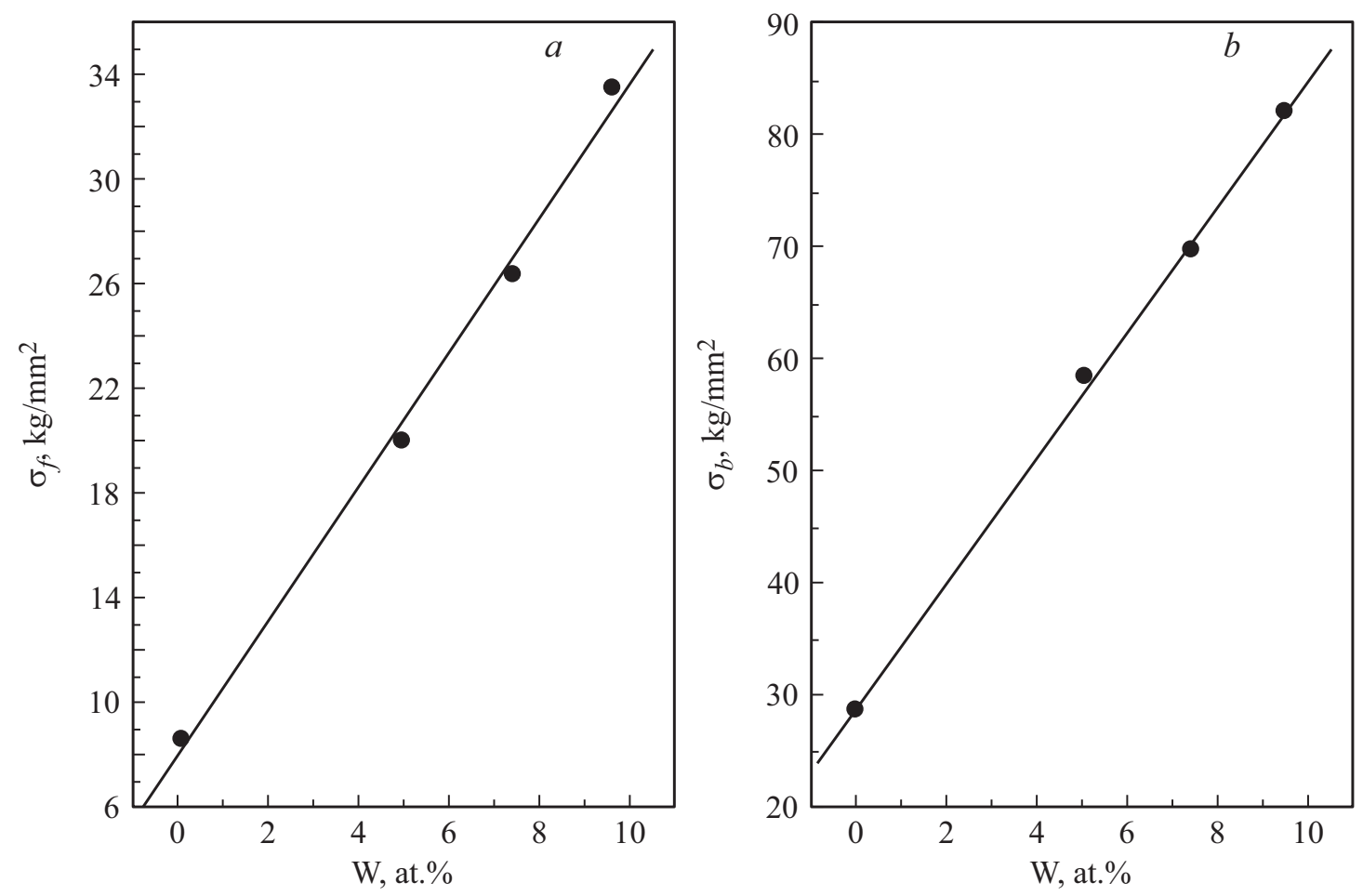

Рис. 2. Зависимость предела текучести $\sigma_{f}(a)$ и предела прочности $\sigma_{b}(b)$ от концентрации вольфрама в сплаве $\mathrm{Ni}-\mathrm{W}$ различного состава.

\section{3. Осаждение TiN}

Покрытия на основе TiN получали методом конденсации титана из плазменного потока, генерируемого дугой низкого давления [27,28]. Данный метод позволяет проводить обработку поверхности образцов высокоэнергетическими ионами, которые экстрагируются из плазмы в результате подачи на образец высокого отрицательного потенциала $U$, что обеспечивает эффективное очищение поверхностного слоя от загрязнений, обусловливая тем самым высокие адгезионные свойства покрытий.

В экспериментах по осаждению TiN подложками служили ленточные образцы сплавов $\mathrm{Ni}-5$ at.\% $\mathrm{W}$ и $\mathrm{Ni}-9.5$ at.\% W. Образцы помещали в центр вакуумной камеры, а в рабочий объем вводился азот $\mathrm{N}_{2}$. В качестве катодного материала использовали титан марки ВТ-1.

Установлено [22,24], что оптимальными параметрами эксперимента по осаждению $\mathrm{TiN}$ покрытий являются: отрицательный потенциал подложки $U=300 \mathrm{~V}$; ток дуги $I=80 \mathrm{~A}$; давление азота в камере $p_{\mathrm{N}}=$ $=1.8 \cdot 10^{-2}$ Torr. Время осаждения варьировало в диапазоне $\tau_{\mathrm{TiN}}=60-900 \mathrm{~s}$.

\section{4. Определение толщины покрытия TiN}

Используемый в настоящей работе метод определения толщины слоев TiN построен на измерении интенсивности рентгеновских интерференций от материала плоской подложки под воздействием покрытия [29]. Интенсивность пучка, отраженного от кристаллической плоскости (hkl) образца с покрытием толщиной $h$ и линейным коэффициентом поглощения $\mu_{\mathrm{TiN}}=880 \mathrm{~cm}^{-1}$, имеет вид

$$
I_{\mathrm{hkl}}^{\mathrm{NiW}}(h)=I_{\mathrm{hkl}}^{\mathrm{NiW}}(0) \exp \left(-2 \mu_{\mathrm{TiN}} h / \cos (\theta)\right),
$$

где $I_{\mathrm{hkl}}^{\mathrm{NiW}}(h)$ - интенсивность пучка, отраженного от образца с покрытием, $I_{\mathrm{hkl}}^{\mathrm{NiW}}(0)$ - интенсивность пучка, отраженного от образца без покрытия, $\mu_{\mathrm{TiN}}-$ линейный коэффициент поглощения $\mathrm{TiN}, h-$ толщина слоя покрытия, $\theta-$ угол отражения.

Формула (1) и математическое моделирование зависимости относительной интенсивности рентгеновских интерференций от времени осаждения $\tau_{\mathrm{TiN}}$, дают возможность определять толщину покрытия независимо от состава сплава. Во избежание ошибок при определении толщины, связанных с возможностью протекания процессов текстурообразования в подложке, эксперименты проводились одновременно на двух образцах, находящихся в идентичных условиях. В настоящей работе тестовым объектом для определения $h_{\mathrm{TiN}}$ служил ленточный образец на основе сплава $\mathrm{Ni}-5$ at.\% $\mathrm{W}$ co стабильной кубической текстурой рекристаллизации.

\section{5. Метод исследования текстуры}

Стоит отметить, что в ленте из сплава $\mathrm{Ni}-9.5 \mathrm{at} \% \mathrm{~W}$ реализуются достаточно сильные текстуры. В этой связи помимо применения классических методов построения полюсных фигур [30] был развит алгоритм исследования 
плоскостной текстуры, который заключается в построении и анализе углового распределения интенсивностей рентгеновских интерференций от кристаллографических плоскостей [24]. Очевидно, что для идеальной кубической текстуры интенсивность дифракционных линий не должна зависеть от угла поворота в собственной плоскости образца. В настоящей работе проводили измерения углового распределения интенсивности дифракции от кристаллографических плоскостей типа (h00) ГЦК решеток подложки и покрытия. Для вычислений использовалось $n=23$ степени свободы, а в качестве критерия достоверности гипотезы о реализации совершенной кубической текстуры был выбран известный статистический метод $\chi^{2}[31]$.

\section{2. Результаты исследования}

Ниже приводятся экспериментальные данные, полученные в ходе настоящей работы: общий характер дифракционной картины (разд. 2.1), текстурные явления (разд. 2.2), изменение параметров решетки в зависимости от толщины покрытия (разд. 2.3).

\section{1. Общий характер дифракционной картины}

На рис. 3 приведен набор дифрактограмм ленточных образцов $\mathrm{Ni}-5$ at.\% W с покрытием TiN, на которых наблюдается две системы дифракционных линий: от ГЦК решеток сплава $\mathrm{Ni}-5$ at.\% W и соединения TiN типа $\mathrm{NaCl}$. Для обеих фаз двухкомпонентной системы $\mathrm{Ni}-5$ at.\% W/TiN характерно наличие кубической текстуры типа $\{001\}\langle 100\rangle$. Очевидно, что рост слоя TiN происходит по схеме $\{200\}_{\mathrm{NiW}} \|\{200\}_{\mathrm{TiN}}$.

C увеличением времени нанесения покрытия при постоянном давлении азота $\left(p_{\mathrm{N}}=1.8 \cdot 10^{-2}\right.$ Torr $)$ можно

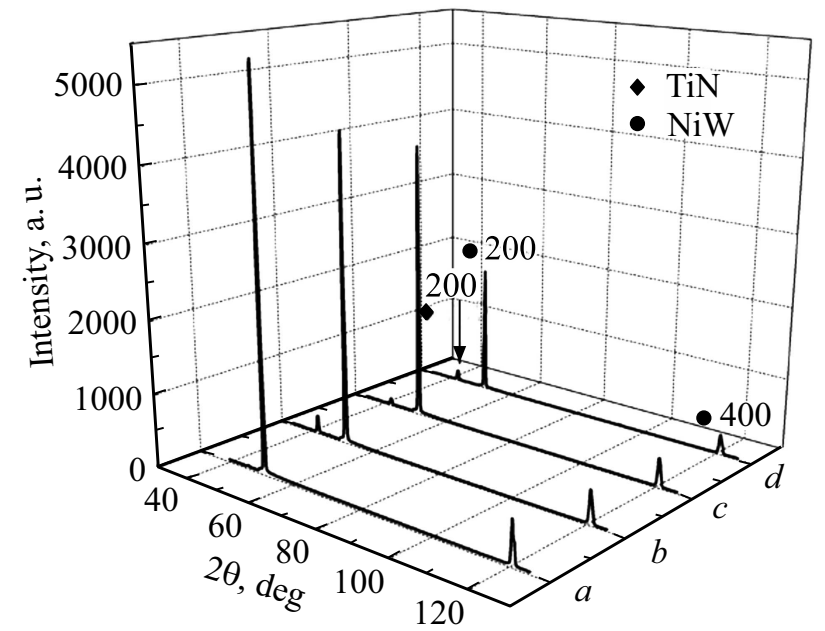

Рис. 3. Рентгенограммы образцов ленты на основе сплава $\mathrm{Ni}-5$ at.\% $\mathrm{W}$ с покрытием TiN различной толщины $h_{\mathrm{TiN}}$ : $a-0, b-\sim 1, c-\sim 2, d-\sim 4 \mu \mathrm{m}$ (толщина ленты $h_{\mathrm{NiW}}=100 \mu \mathrm{m}$; давление азота $p_{N}=1.8 \cdot 10^{-2}$ Torr; $U_{n}=-300 \mathrm{~V}$; ток дуги $I=80 \mathrm{~A}$ ).

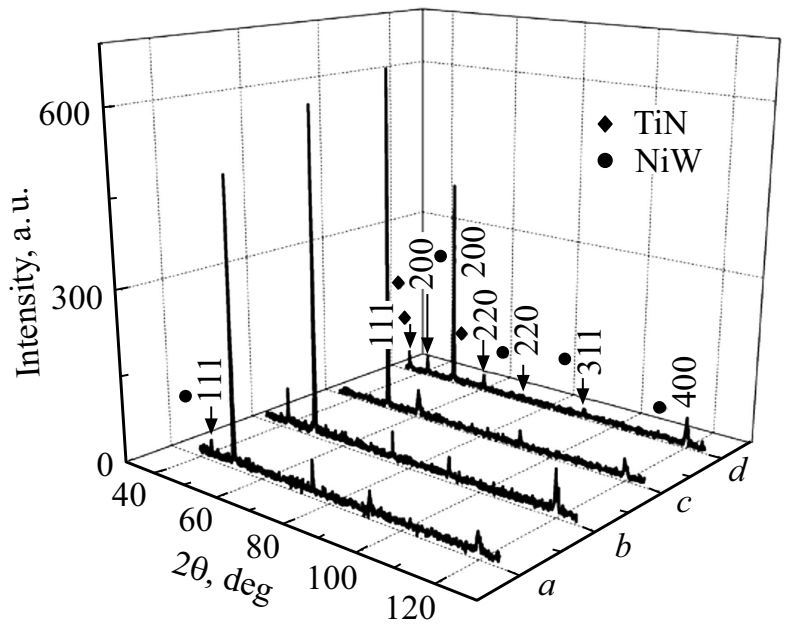

Рис. 4. То же, что на рис. 3 , но для образцов ленты на основе сплава $\mathrm{Ni}-9.5$ at.\% W.

наблюдать уменьшение интенсивности линий подложки, обусловленное ослаблением пучка рентгеновского излучения при прохождении сквозь вещество образца. Этот эффект хорошо описывается уравнением (1) и, как было упомянуто выше, дает возможность воспользоваться инструментом определения толщины покрытия.

Для образцов Ni-9.5 at.\% W c TiN покрытием наблюдается иная картина (рис. 4). В системе $\mathrm{Ni}-9.5$ at.\% W/TiN имеет место аномальный эффект перераспределения относительной интенсивности дифракции на кристаллографических плоскостях подложки, который приводит к усилению интенсивности дифракционных пиков типа $(\mathrm{h} 00)_{\mathrm{NiW}}$.

Следует подчеркнуть, что максимальная интенсивность дифракционного рефлекса, соответствующего кубической плоскости $(200)_{\mathrm{NiW}}$, наблюдается при $h_{\mathrm{TiN}} \sim 2 \mu \mathrm{m}$, в то время как интенсивность пиков $(220)_{\mathrm{NiW}}$ и $(311)_{\mathrm{NiW}}$ уменьшается. В слое TiN при толщине покрытия $h_{\mathrm{TiN}} \sim 1 \mu \mathrm{m}$ присутствует интенсивный дифракционный пик от кубической плоскости $(200)_{\mathrm{TiN}}$ решетки TiN. Дальнейшее увеличение толщины слоя покрытия приводит к появлению пиков $(111)_{\mathrm{TiN}}$ и $(220)_{\mathrm{TiN}}$, указывающих на размытие кубической текстуры нитрида титана.

\section{2. Текстура лент-подложек из сплавов $\mathrm{Ni}-\mathrm{W}$ и слоев TiN}

В качестве примера на рис. 5 приведены типичные картины (круговые диаграммы) углового распределения интенсивности дифракции на плоскостях (200) для обеих компонент системы $\mathrm{Ni}-\mathrm{W} / \mathrm{TiN}$ при $h_{\mathrm{TiN}}=1 \mu \mathrm{m}$. Характер распределения подтверждает наличие эффектов формирования кубической текстуры в ленте на основе сплава $\mathrm{Ni}-5$ at.\% W и слое TiN (рис. 5, $a, b)$. Диаграммы, представленные на рис. $5, a^{\prime}, b^{\prime}$, однозначно указывают 

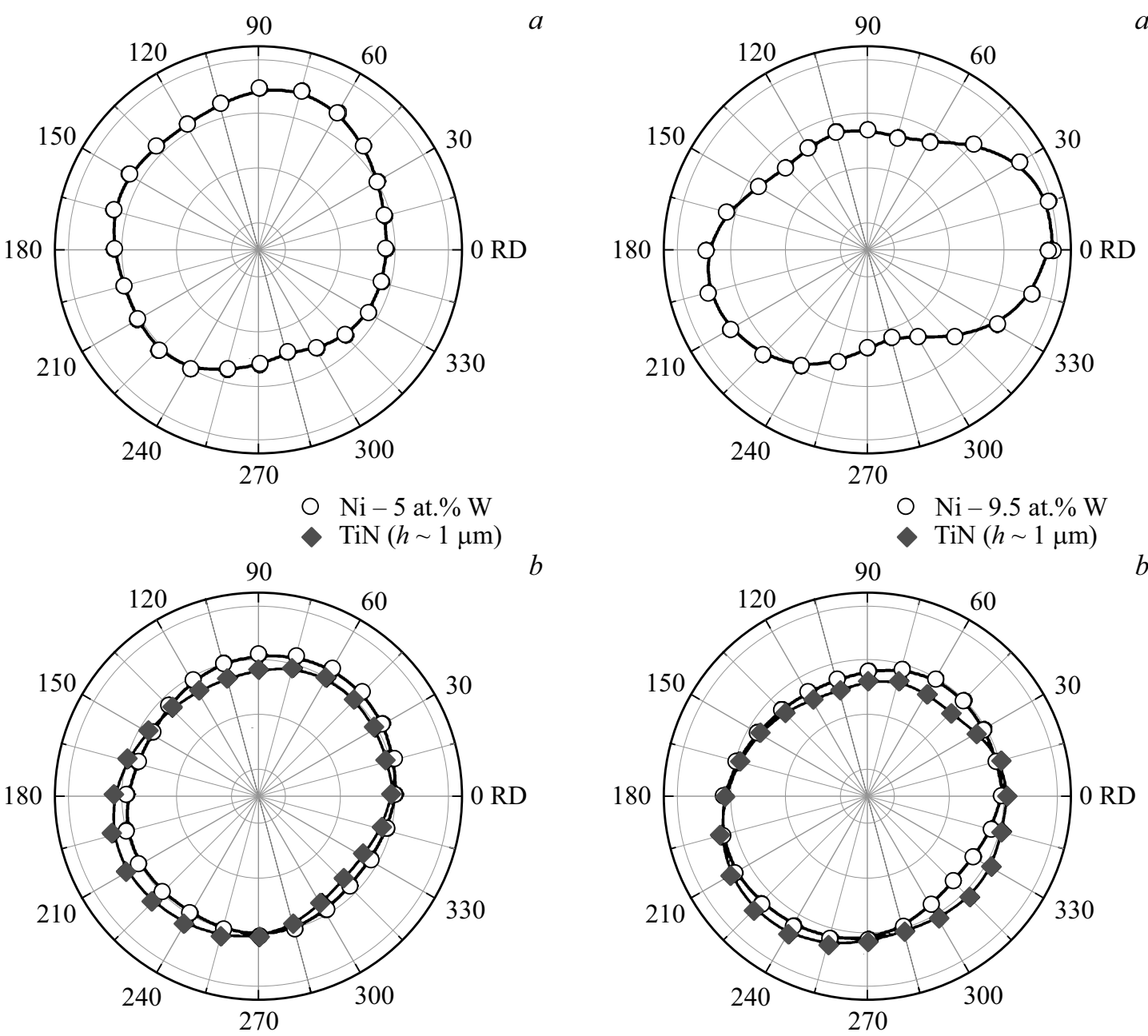

$a^{\prime}$

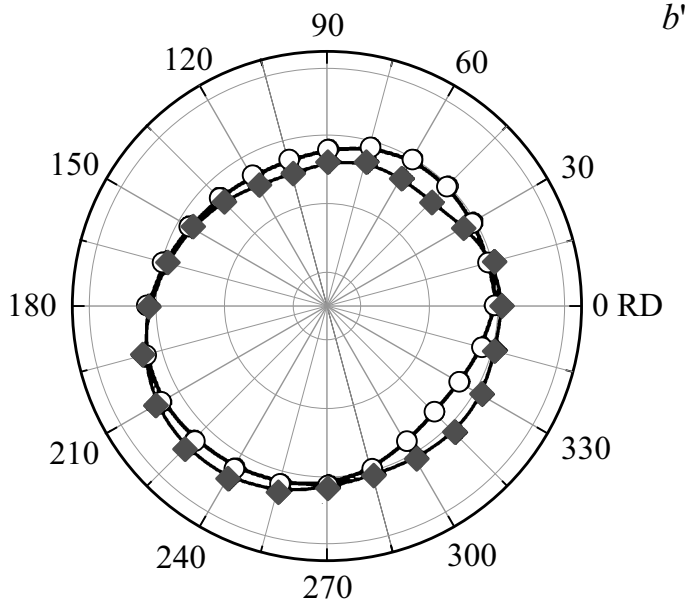

Рис. 5. Диаграммы углового распределения дифракции от кубической плоскости (200): $a$ - образец Ni-5at.\% W без покрытия; $b$ - образец Ni-5 at.\% W с покрытием TiN; $a$ - образец Ni-9.5 at.\% W без покрытия; $b$ - образец Ni-9.5 at\% W с покрытием TiN.

на протекание процессов эволюции текстуры подложки из сплава $\mathrm{Ni}-9.5$ at.\% W под воздействием покрытия.

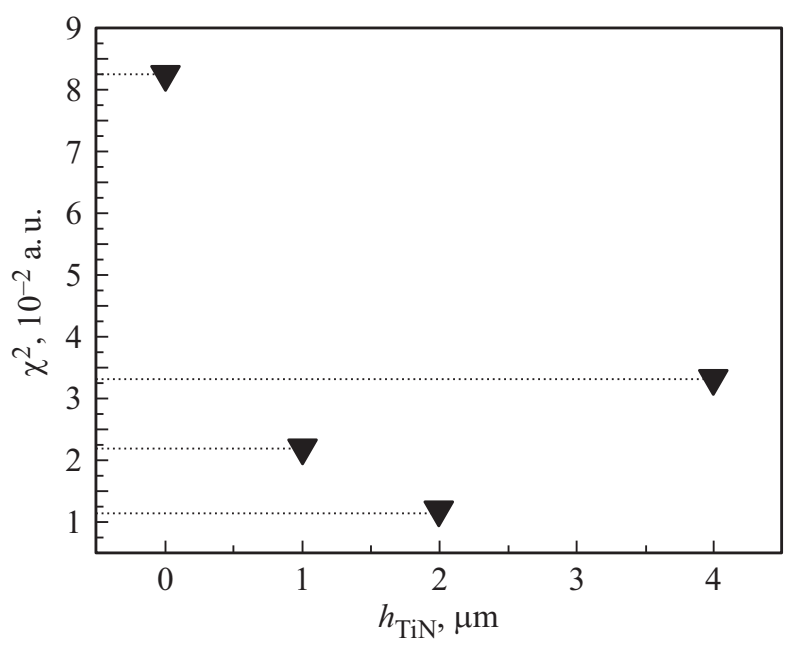

Рис. 6. Значения статистического параметра $\chi^{2}$ в случае подложки из сплава $\mathrm{Ni}-9.5$ at.\% W в зависимости от толщины покрытия TiN.
Поскольку наибольший интерес представляет изучение динамики процессов текстурообразования в системе $\mathrm{Ni}-9.5$ at.\% W/TiN, на рис. 6 приведены значения статистического параметра $\chi^{2}$ для подложки $\mathrm{Ni}-9.5$ at.\% W в зависимости от толщины покрытия $h_{\mathrm{TiN}}$. При $h_{\mathrm{TiN}} \sim 2 \mu \mathrm{m}$ параметр $\chi^{2}$ принимает минимальное значение. Необходимо отметить, что максимальная степень кубической текстуры TiN $\left(\chi^{2}=0.009\right)$ в системе $\mathrm{Ni}-9.5$ at.\% W/TiN имеет место при $h_{\mathrm{TiN}} \sim 1 \mu \mathrm{m}$.

\section{3. Параметры кристаллических решеток лент на основе сплавов $\mathrm{Ni}-5$ at.\% W и Ni-9.5 at.\% W и покрытий TiN}

Для установления природы процессов текстурообразования в тонкослойных системах изучалась динамика изменения параметров решетки в системе „подложка-покрытие“.

Зависимости параметра решетки TiN от толщины покрытия, осажденного на подложки $\mathrm{Ni}-\mathrm{W}$ с разным содержанием W, представлены на рис. 7. С ростом тол- 

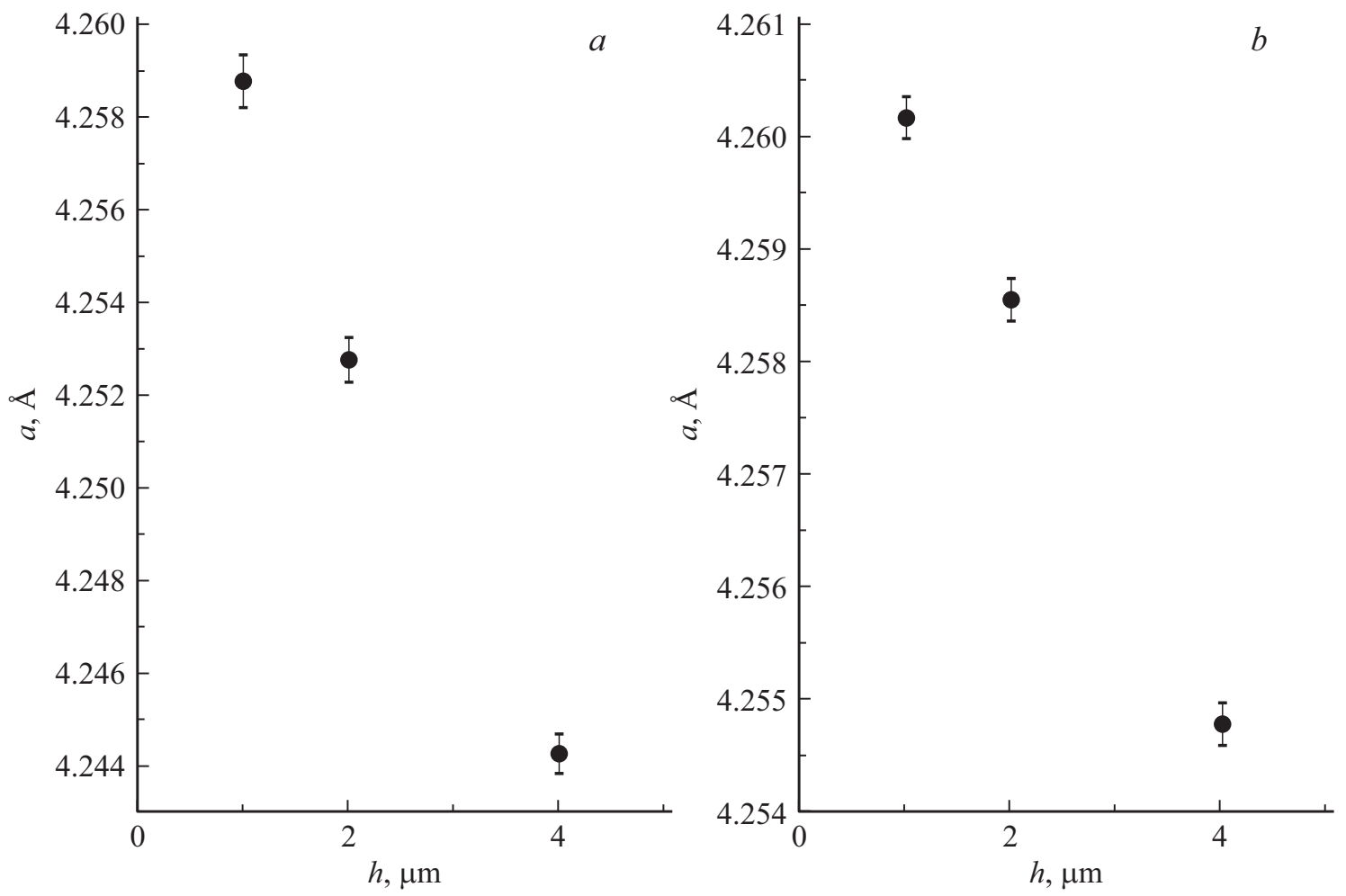

Рис. 7. Изменение величины параметра кристаллической решетки слоя $\mathrm{TiN}$, нанесенного на подложку из сплава $\mathrm{Ni}-5 \mathrm{at} . \% \mathrm{~W}(a)$ и Ni-9.5 at.\% W (b) в зависимости от толщины покрытия. Параметр решетки $a_{\text {TiN }}$ опредяляли по дифракционной линии $(200)$ TiN.
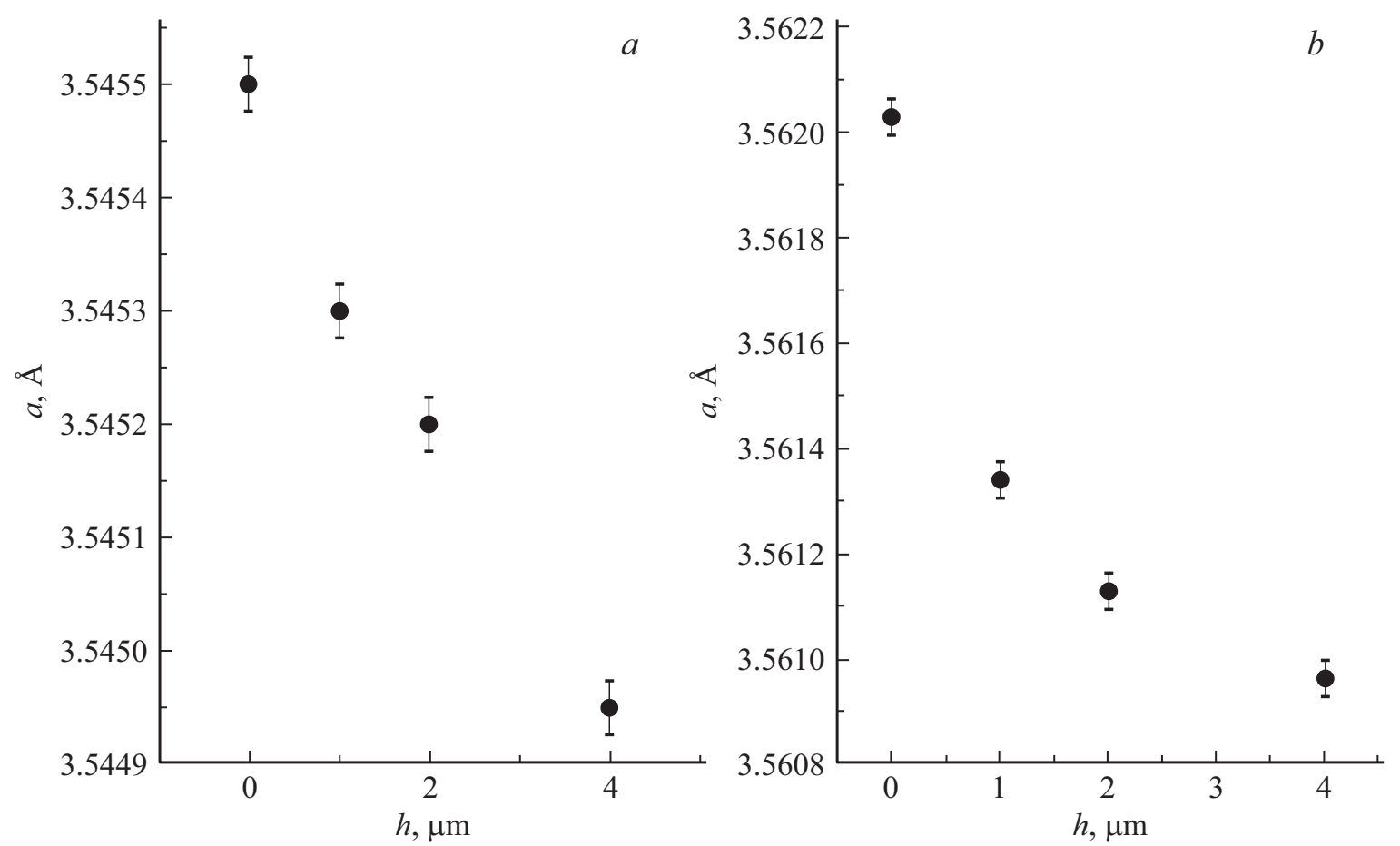

Рис. 8. Зависимость параметров кристаллической решетки подложки на основе сплава $\mathrm{Ni}-5$ at.\% $\mathrm{W}(a)$ и $\mathrm{Ni}-9.5 \mathrm{at} . \% \mathrm{~W}(b)$ от толщины покрытия TiN. Параметр решетки $a_{\mathrm{Ni}-\mathrm{W}}$ опредяляли по дифракционной линии $(400)_{\mathrm{Ni}-\mathrm{W}}$.

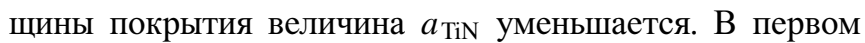
приближении обе зависимости $a_{\text {TiN }}(h)$ носят линейный характер, а максимальный эффект изменения $a_{\mathrm{TiN}}$ наблюдается в системе Ni-5at.\% W/TiN. 
На рис. 8 приведены кривые изменения параметров кристаллической решетки $a_{\mathrm{Ni}-\mathrm{W}}$ (измеренных в направлении, поперечном к направлению прокатки) лент $\mathrm{Ni}-5$ at.\% $\%$ (рис. 8, $a$ ) и $\mathrm{Ni}-9.5$ at.\% $\mathrm{W}$ (рис. $8, b$ ) в зависимости от толщины покрытия. Можно видеть, что с ростом $h_{\mathrm{TiN}}$ величины $a_{\mathrm{Ni}-\mathrm{W}}$ уменьшаются для образцов обоих составов. При этом в случае $\mathrm{Ni}-9.5$ at.\% W данный эффект выражается значительно

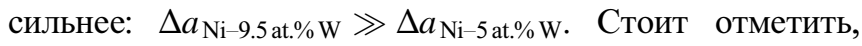
что практически линейный ход (коэффициент корреляции $\left.R^{2}=99.99\right)$ зависимости $a_{\mathrm{Ni}-5 \mathrm{at} . \% \mathrm{~W}}\left(h_{\mathrm{TiN}}\right)$ сменяется на экспоненциальный $\left(R^{2}=99.98\right)$ для $a_{\mathrm{Ni}-9.5 \text { at.\% }}\left(h_{\mathrm{TiN}}\right)$.

\section{3. Обсуждение результатов}

Предметом обсуждения в настоящей работе является совокупность обнаруженных эффектов, связанных с особенностями формирования текстуры в тонкослойных системах $\mathrm{Ni}-\mathrm{W} / \mathrm{TiN}$.

Из рентгенограмм, приведенных на рис. 3, и диаграмм углового распределения интенсивности дифракции рентгеновского излучения от кубической плоскости (200) подложки и покрытия (рис. $5, a, b$ ) следует, что осаждение $\mathrm{TiN}$ не приводит к существенным изменениям текстуры в ленте-подложке из сплава $\mathrm{Ni}-5 \mathrm{at} . \% \mathrm{~W}$, изначально обладающей сильной кубической текстурой рекристаллизации.

Обнаруженный эффект перераспределения относительной интенсивности дифракционных линий подложки (рис. 4) из сплава $\mathrm{Ni}-9.5$ at.\% W в результате осаждения покрытия описать с помощью классических законов поглощения не удается. Единственной причиной аномального поведения интенсивности дифракционных пиков подложки при увеличении толщины покрытия может служить переориентация кристаллографических плоскостей в лабораторной системе координат. Это означает, что в тонкослойной системе $\mathrm{Ni}-9.5$ at.\% W/TiN имеет место эффект текстурообразования в подложке под действием покрытия („контр-эпитаксия“). Насколько известно подобный эффект ранее не наблюдался. Для объяснения этого явления может быть предложен следующий механизм. С ростом толщины покрытия величина

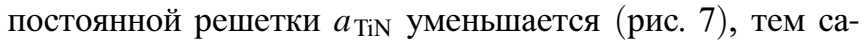
мым обнаруживая тенденцию к сближению параметров кристаллических решеток обеих компонент системы. Очевидно, что величина $a_{\mathrm{Ni}-\mathrm{W}}$ в направлении, перпендикулярном к направлению прокатки, должна уменьшаться с ростом $h_{\mathrm{TiN}}$. Однако в поведении зависимостей параметров решеток $a_{\mathrm{Ni}-5 \mathrm{at} . \% \mathrm{~W}}\left(h_{\mathrm{TiN}}\right)$ и $a_{\mathrm{Ni}-9.5 \mathrm{at.} \% \mathrm{~W}}\left(h_{\mathrm{TiN}}\right)$ наблюдаются качественные и количественные различия (рис. 8). Характер изменения параметра кристалличе-

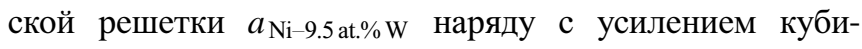
ческой текстуры подложки и покрытия в диапазоне $h_{\mathrm{TiN}} \sim 1-2 \mu \mathrm{m}$ дает основания полагать, что в системе Ni-9.5 at.\% W/TiN происходит повышение энергии дефектов упаковки $E_{s f}$, вызванное появлением внутренних напряжений на границе раздела двухслойной системы $\mathrm{Ni}-\mathrm{W} / \mathrm{TiN}$.

Поскольку практическое значение для создания $2 \mathrm{G}$ HTS имеет эффект образования сильной биаксиальной текстуры $\mathrm{TiN}$, наибольший интерес представляет тот факт, что совершенная эпитаксиальная кубическая текстура покрытия наблюдается в тонких слоях. При $h_{\mathrm{TiN}} \gtrsim 1 \mu \mathrm{m}$ в слое TiN начинает преобладать текстура роста в направлении $\langle 111\rangle$.

\section{Заключение}

Основное содержание настоящей работы составляет изучение структурных эффектов в обеих компонентах тонкопленочной системы $\mathrm{Ni}-\mathrm{W} / \mathrm{TiN}$. Фактически рассматривались две ситуации: 1) осаждение TiN на заведомо текстурированную подложку на основе ферромагнитного сплава $\mathrm{Ni}-5$ at.\% W; 2) нанесение TiN на подложку из парамагнитного сплава $\mathrm{Ni}-9.5$ at.\% W, в которой формирование кубической текстуры рекристаллизации затруднено в связи с низким значеним $E_{s}$.

В первом случае был достигнут ожидаемый результат, заключавшийся в образовании кубической текстуры в слоях TiN.

При осаждении покрытия на ленту-подложку из $\mathrm{Ni}-9.5$ at.\% W впервые обнаружен эффект контрэпитаксии: усиление текстуры подложки под воздействием покрытия. При этом в системе Ni-9.5 at.\% W/TiN с ростом толщины покрытия происходит существенное изменение параметра кристаллической решетки $a_{\mathrm{Ni}-\mathrm{W}}$.

Кроме того, процессы текстурообразования в подложке и покрытии разнесены по толщине. Максимальная степень кубической текстуры TiN реализуется в достаточно тонких слоях толщиной $h_{\mathrm{TiN}} \sim 1 \mu \mathrm{m}$, тогда как кубическая текстура $\mathrm{Ni}-9.5$ at.\% W формируется при $h_{\text {TiN }} \sim 2 \mu \mathrm{m}$. Дальнейшее повышение толщины слоя покрытия приводит к ухудшению текстуры обеих компонент системы Ni-9.5 at.\% W/TiN.

Результаты исследования указывают на то, что ленты из парамагнитного $\mathrm{Ni}-9.5$ at.\% W c TiN покрытием могут рассматриваться в качестве перспективных подложек при создании эффективных 2G HTS сверхпроводников.

Авторы выражают благодарность С.А. Леонову, Ю.Н. Шахову и В.В. Деревянко за помощь в проведении экспериментов.

\section{Список литературы}

[1] Goyal A., Norton D.P., Budai J.D., Paranthaman M., Specht E.D., Kroeger D.M. // Appl. Phys. Lett. 1996. Vol. 69. P. 1795-1797. DOI: $10.1063 / 1.117489$

[2] Seeber B. Power Applications of Superconductivity: Handbook of Applied Superconductivity. Bristol, UK: Inst. Physics, 1998. P. 1485-1756. 
[3] Malozemoff A.P., Yamada Y. Coated conductor: second generation HTS wire. In 100 Years of Superconductivity / Ed. by H. Rogalla, P. Kes. N. Y.: Taylor \& Francis, 2011. P. 689-702.

[4] Rupich M.W., Li X., Thieme C., Sathyamurthy S., Fleshler S. et al. // Supercond. Sci. Technol. 2010. Vol. 23. P. 014015. DOI: 10.1088/0953-2048/23/1/014015

[5] Gaitzsch U., Hanisch J., Huhne R., Rodig C., Freudenberger J., Holzapfel B., Schultz L. // Supercond. Sci. Technol. 2013. Vol. 26. P. 085024. DOI: 10.1088/0953-2048/26/8/085024

[6] Gaitzsch U., Eickemeyer J., Rodig Ch., Freudenberger J., Holzapfel B., Schultz L. // Scr. Mater. 2010. Vol. 62. P. 512515. DOI: $10.1016 /$ j.scriptamat.2009.12.030

[7] Zhao Y., Suo H.-L., Liu M., He D., Zhang Y.-X., Ma L., Zhou M.-L. // Acta Mater. 2007. Vol. 55. N 8. P. 2609-2614. DOI: 10.1016/j.actamat.2007.01.001

[8] Selvamanickam V., Chen Y., Kesgin I. et al. // IEEE Tr. Appl. Supercond. Vol. 21. N 3. P. 3049-3054. DOI: 10.1109/TASC.2011.2107310

[9] Stewart E., Bhuiyan M.S., Sathyamurthy S., Paranthaman M. // Mater. Res. Bull. 2006. Vol. 41. N 6. P. 1063-1068. DOI: 10.1016/j.materresbull.2005.11.015

[10] Mathias V., Rowley E.J., Coulter Y. et al. // Supercond. Sci. Technol. 2010. Vol. 23. P. 014018. DOI: $10.1088 / 0953-2048 / 23 / 1 / 014018$

[11] Hühne R., Gärtner R., Oswald S., Schultz L., Holzapfel B. // Physica C. 2011. Vol. 471. N 21-22. P. 966-969.

DOI:10.1016/j.physc.2011.05.101

[12] Hühne R., Fähler S., Holzapfel B. // Appl. Phys. Lett. 2004. Vol. 85. P. 2744. DOI: $10.1063 / 1.1802385$

[13] Hühne R., Güth K., Kidszun M., Kaltofen R., Schultz L., Holzapfel B. // J. Phys. D: Appl. Phys. 2008. Vol. 41. P. 245404. DOI: $10.1088 / 0022-3727 / 41 / 24 / 245404$

[14] Zhang Y., Yan P., Wu Z., Zhang P. // Rare Metals. 2005. Vol. 24. N 4. P. 370-375.

[15] Akkaya S.S., Vasyliev V.V., Reshetnyak E.N., Kazmanl K., Solak N., Strel'nitskij V.E., Ürgen M. // Surf. Coat. Technology. 2013. Vol. 236. P. 332-340. DOI: 10.1016/j.surfcoat.2013.10.009

[16] Eickenmeier J., Huhne R., Guth A., Rodig C., Gaitzsch U., Freudenberger J., Schultz L., Holzapfelet B. // Supercond. Sci. Technol. 2010. Vol. 23. P. 085012. DOI: 10.1088/0953-2048/23/8/085012

[17] Ijodola A.O., Thomson J.R., Goyal A., Thieme C.L.H., Marken K. // Physica C. 2004. Vol. 403. P. 163-171. DOI: $10.1016 /$ j.physc.2003.12.003

[18] Genenko Y.A., Rauh H., Kruger P. // Appl. Phys. Lett. 2011. Vol. 98. P. 152303. DOI: 10.1063/1.3560461

[19] Subramanya Sarma V., Eickemeyer J., Schultz L., Holzapfel B. // Scripta Mater. 2004. Vol. 50. N 7. P. 953-957. DOI: $10.1016 /$ j.scriptamat.2004.01.004

[20] Mohamed F.A., Langdon T.G. // Metall. Trans. A. 1975. Vol. 6. P. 927.

[21] Siegel D.J. // Appl. Phys. Lett. 2005. Vol. 87. P. 121901. DOI: $10.1063 / 1.2051793$

[22] Finkel V.A., Bovda A.M., Leonov S.A. et al. // Funct. Mater. 2012. Vol. 19. N 1. P. 109-115.

[23] Finkel V.A., Derevyanko V.V., Sunhurov M.S., Sukhareva T.V., Shahov Yu.N. // Funct. Mater. 2013. Vol. 20. N 1. P. 103-110. DOI: $10.15407 / \mathrm{fm} 20.01 .103$
[24] Сунгуров М.С., Деревянко В.В., Леонов С.А., Сухарева Т.В., Финкель В.А., Шахов Ю.Н. // Письма в ЖТФ. 2014 T. 40. Вып. 8. C. 47-53. [Sungurov M.S., Derevyanko V.V., Leonov S.A. etal. // Tech. Phys. Lett. 2014. Vol. 40. P. 797-800. DOI: 10.1134/S1063785014090314]

[25] Sunhurov M.S., Leonov S.A., Sukhareva T.V., Derevyanko V.V., Finkel V.A., Shakhov Yu.N. // Funct. Mater. 2017. Vol. 24. N 1. P. $063-067$. DOI: $10.15407 / \mathrm{fm} 24.01 .063$

[26] Zhao Y., Suo H.L., Zhu Y., Liu M. etal. // Supercond. Sci. Technol. 2008. Vol. 21. P. 075003. DOI: $10.1088 / 0953-2048 / 21 / 7 / 075003$

[27] Белоус В.А., Васильев В.В., Лучанинов А.А., Решетняк Е.Н., Стрельницкий В.Е., Толмачева Г.Н., Голтвяница В.С., Голтвяница С.К. // Физическая инженерия поверхности. 2009. Т. 7. Вып. 3. С. 216-222.

[28] Aksenov I., Belokhvostikov A.N., Padalka V.G. et al. // Plasma Phys. Contr. F. 1986. Vol. 28. P. 761. DOI: $10.1088 / 0741-3335 / 28 / 5 / 002$

[29] Aliaj F.R., Syla N., Oettel H., Dilo T. // Surf. Interface Anal. 2017. Vol. 49. N 11. P. 1135-1141. DOI: $10.1002 /$ sia.6292

[30] Бородкина М.M., Спектор Э.Н. // Рентгено-графический анализ текстуры металлов и сплавов. М.: Металлургия, 1981. $272 \mathrm{c}$.

[31] Худсон Д. Статистика для физиков. М.: Мир, 1970. 296 с. 